\title{
A Study on the Application of Fuzzy Information Seeded Region Growing in Brain MRI Tissue Segmentation
}

\author{
Chuin-Mu Wang' and Geng-Cheng Lin ${ }^{2}$ \\ ${ }^{1}$ Department of Computer Science and Information Engineering, National Chin-Yi, University of Technology, Taiping 411, Taiwan \\ ${ }^{2}$ Networks and Communications Group, Advantech Co., Ltd., Neihu 114, Taiwan \\ Correspondence should be addressed to Chuin-Mu Wang; cmwang@ncut.edu.tw
}

Received 25 February 2014; Accepted 4 April 2014; Published 5 May 2014

Academic Editor: Her-Terng Yau

Copyright (c) 2014 C.-M. Wang and G.-C. Lin. This is an open access article distributed under the Creative Commons Attribution License, which permits unrestricted use, distribution, and reproduction in any medium, provided the original work is properly cited.

After long-term clinical trials, MRI has been proven to be used in humans harmlessly, and it is popularly used in medical diagnosis. Although MR is highly sensitive, it provides abundant organization information. Therefore, how to transform the multi-spectral images which is easier to be used for doctor's clinical diagnosis. In this thesis, the fuzzy bidirectional edge detection method is used to solve conventional SRG problem of growing order in the initial seed stages. In order to overcome the problems of the different regions, although it is the same Euclidean distance for region growing and merging process stages, we present the peak detection method to improve them. The standard deviation target generation process (SDTGP) is applied to guarantee the regions merging process does not cause over- or undersegmentation. Experimental results reveal that FISRG segments a multispectral MR image much more effectively than FAST and $K$-means.

\section{Introduction}

Currently there are several common detection technologies like digitized X-ray photography (digital radiography, $\mathrm{DR}$ ), fine needle aspiration cytology (fine needle aspiration cytology, FNAC), ultrasound testing (ultrasonic testing, UT), computed tomography scan (computed tomography, CT), magnetic resonance imaging techniques (magnetic resonance imaging, MRI), and so forth. In 1977, a brand new and has no medical radiation damage detection techniques (magnetic resonance imaging) in response born. MRI (magnetic resonance imaging) is not required under the developer and has been able to show a clearer tissue contrast image, with no ionizing radiation, noninvasiveness, and other characteristics, and is widely used in medical diagnosis and follow-up. Currently the use of MRI in the medical is very broad, such as tumor component analysis, stroke diagnosis, drawing blood, and spinal magnetic resonance diagrams. With advances in technology and medical knowledge accumulated experience, radiology medical research led to clinical diagnosis towards the era of digital technology, the medical image processing and compression techniques, in addition to reducing the burden of health care for pathologic interpretation and also enhancing the clinical diagnosis accuracy and objectivity, so the medical imaging related research not only improves quality of care, but also reflects its overall social value.

Currently, there are five methods adopted for segmentation: cluster-based segmentation, using common classification approaches to separate similar image data into clusters [1-3]; boundary-based segmentation, applied to calculate the edge gradient difference of each cluster $[4,5]$; thresholdbased segmentation, separating an image according to a threshold value [6]; region-based segmentation, whose greatest difference from cluster-based segmentation is the merging of small areas into blocks in accordance with the similarity of image data [7]; and hybrid-based segmentation, performed with both the edge gradient and region similarity taken into consideration.

Seeded region growing (SRG) is a hybrid-based segmentation algorithm put forward by Adams and Bischof in 


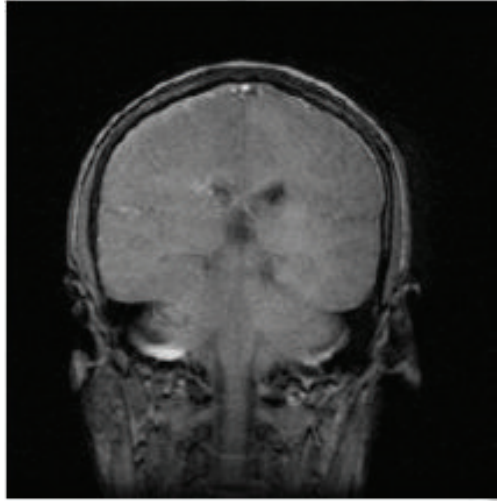

(a)

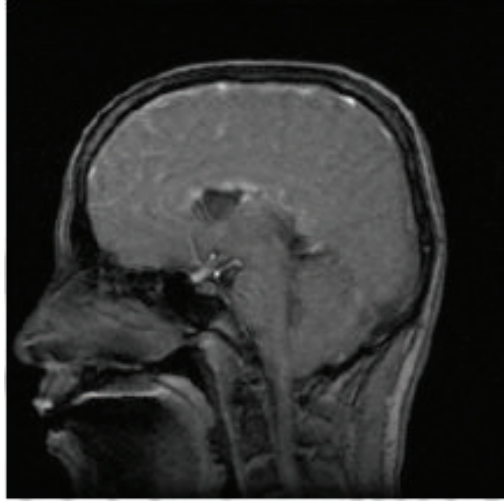

(b)

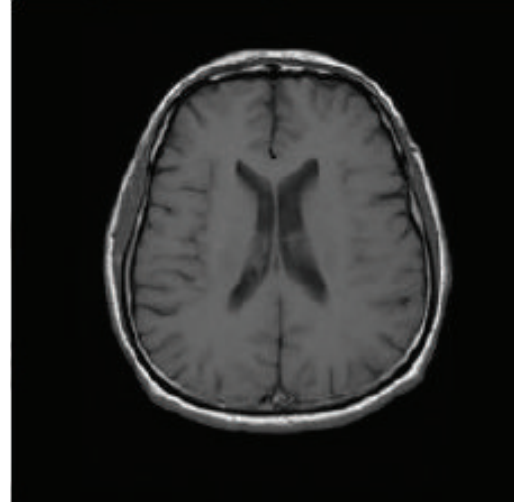

(c)

FIGURE 1: Three plane images of a real brain: (a) coronal plane, (b) sagittal plane, and (c) axial plane.

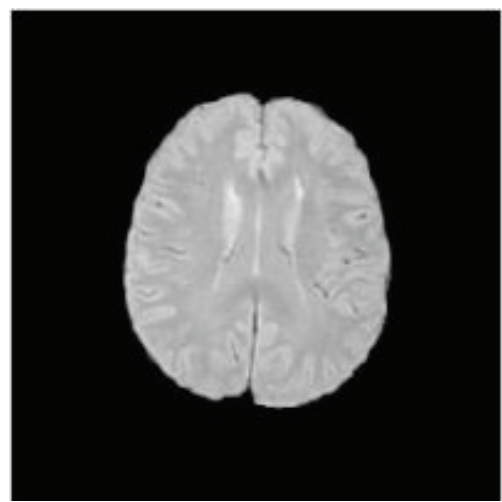

(a)

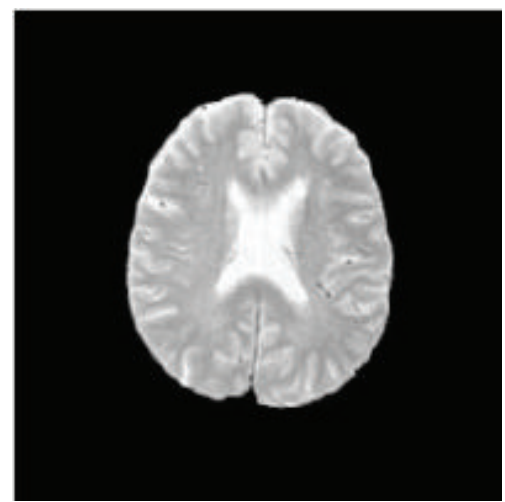

(b)

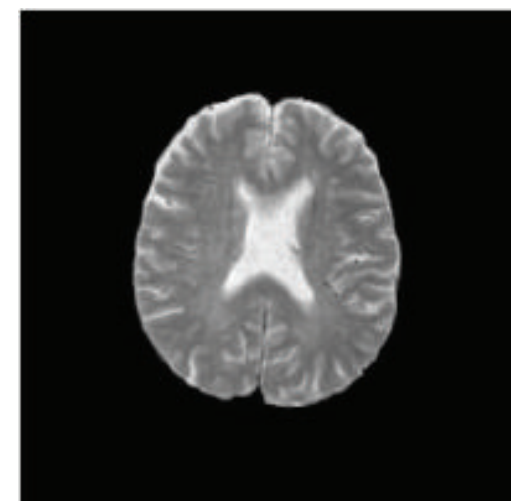

(c)

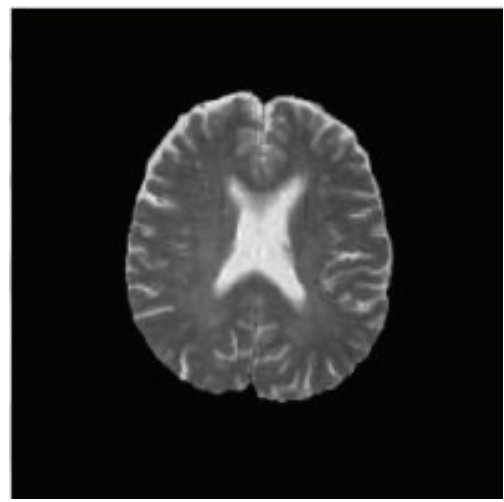

(d)

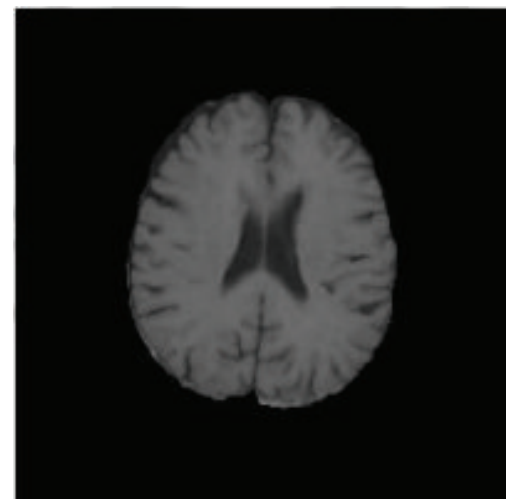

(e)

Figure 2: Real brain MR images. (a) Band1, (b) Band2, (c) Band3, (d) Band4, and (e) Band5.

1994 [8]. When this algorithm is applied, a seed is first sown in an area of interest. Then starting from the initial seed, pixels of higher similarity are merged to grow. When a region comes in contact with other regions during the growing process, this region will stop growing toward that direction and turns toward another direction to ensure there is no overlap between regions [9].

\section{Multispectrum Images and Simulated Images}

MRI has coronal, sagittal, and axial sections of three scan directions. In this paper we use the coronal section images, as shown in Figure 1. In accordance with the same parameters, Figure 2 lists the five brain MR images and the same section. 


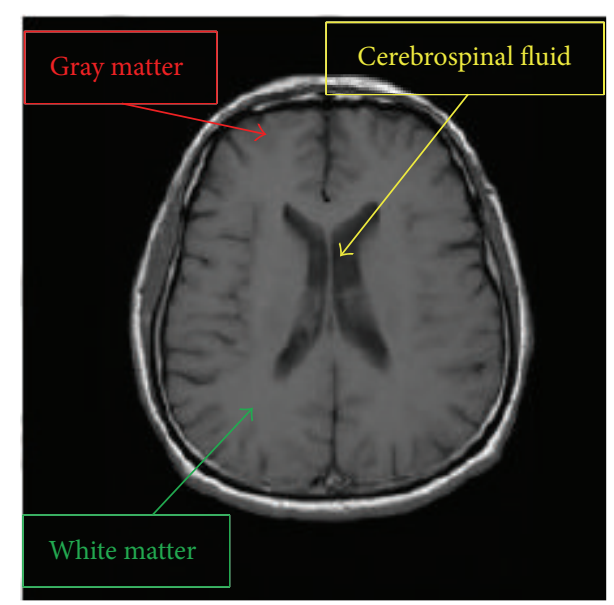

FIgURE 3: Distribution of three main brain tissues.

\begin{tabular}{|c|c|c|}
\hline$X_{1}$ & $X_{2}$ & $X_{3}$ \\
\hline$X_{4}$ & $X_{5}$ & $X_{6}$ \\
\hline$X_{7}$ & $X_{8}$ & $X_{9}$ \\
\hline
\end{tabular}

\begin{tabular}{|l|l|l|}
\hline$X_{x-1, y-1}$ & $X_{x-1, y}$ & $X_{x-1, y+1}$ \\
\hline$X_{x, y-1}$ & $X_{x, y}$ & $X_{x, y+1}$ \\
\hline$X_{x+1, y-1}$ & $X_{x+1, y}$ & $X_{x+1, y+1}$ \\
\hline
\end{tabular}

Figure 4: Arrangement of the selected pixel and neighboring pixels under mask.

PD weight spectrum is the main image of Band1 (proton density image, PDI) (the orientation parameter: TR/TE = $2500 \mathrm{~ms} / 25 \mathrm{~ms}$ ). Band2, Band3, and Band 4 are based on the weight class of T2 spectrum (T2 weighted image, T2WI) (the orientation parameters: TE $/ \mathrm{TE}=2500 \mathrm{~ms} / 50 \mathrm{~ms}$, TR $/ \mathrm{TE}=$ $2500 \mathrm{~ms} / 75 \mathrm{~ms}$, and TR/TE $=2500 \mathrm{~ms} / 100 \mathrm{~ms})$. and Band5 is based on T1 weighted images of the spectrum-based (T1 weighted image, T1WI) (the orientation parameter: TR/TE = $500 \mathrm{~ms} / 11.9 \mathrm{~ms}$ ). The classification of the brain is three main objectives of GM (gray matter), WM, and CSF (cerebral spinal fluid), shown in Figure 3.

\section{Fuzzy Information Seeded Region Growing}

3.1. Initial Seed Selection. We use fuzzy edge detection and fuzzy similarity computation to calculate and select appropriate initial seeds $[10,11]$. First, a $3 \times 3$ mask is applied to calculate the vector of each pixel in the image and the rate of neighboring pixels falling on the edge, as shown in Figure 4.

Directional edge can be broadly divided into four (90, $0,45,135)$, as calculated for each pixel is the edge of the extent; if there is no way considered borderline cases, subsequent connections will result in slight misjudgment occurred degree edge. Therefore, we have established eight masks in Figure 5; all the pixels from the entire image fall on the edge of the level. And the $3 \times 3$ pixels in the mask

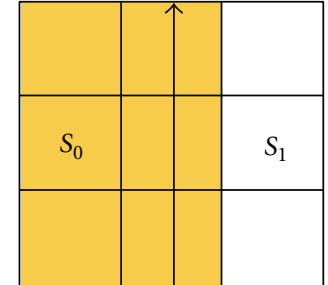

Direction-1

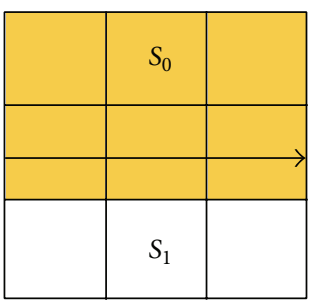

Direction-3

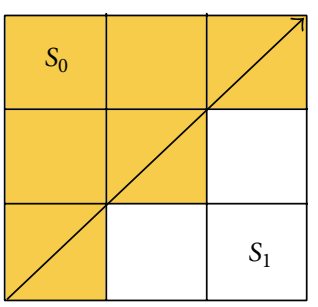

Direction-5

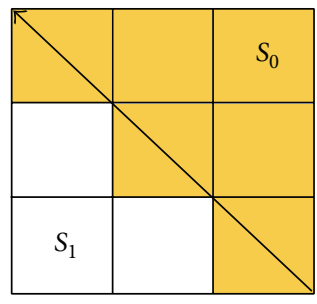

Direction-7

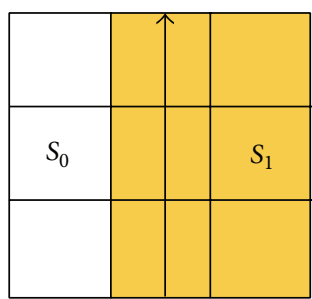

Direction-2

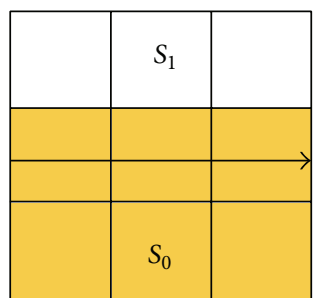

Direction-4

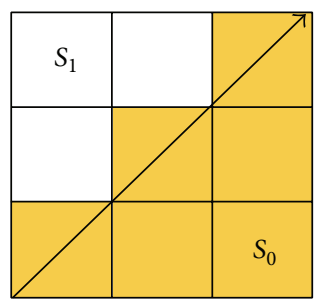

Direction-6

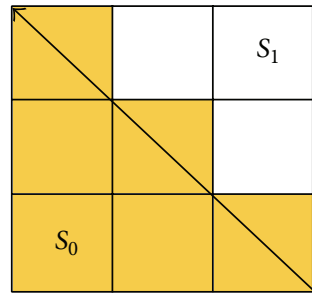

Direction-8
FIGURE 5: Four possible edge directions and their direction mask contrasts.

are divided into two sets, as in the following Direction-1 to -4 descriptions:

90 degrees (forward)

$$
\begin{aligned}
& S_{0}^{1}=\left\{x_{1}, x_{2}, x_{4}, x_{5}, x_{7}, x_{8}\right\}, \\
& S_{1}^{1}=\left\{x_{3}, x_{6}, x_{9}\right\} ;
\end{aligned}
$$

90 degrees (reverse)

$$
\begin{aligned}
& S_{0}^{2}=\left\{x_{2}, x_{3}, x_{5}, x_{6}, x_{8}, x_{9}\right\}, \\
& S_{1}^{2}=\left\{x_{1}, x_{4}, x_{7}\right\} ;
\end{aligned}
$$

0 degrees (forward)

$$
\begin{aligned}
& S_{0}^{3}=\left\{x_{1}, x_{2}, x_{3}, x_{4}, x_{5}, x_{6}\right\}, \\
& S_{1}^{3}=\left\{x_{7}, x_{8}, x_{9}\right\} ;
\end{aligned}
$$


0 degrees (reverse)

$$
\begin{aligned}
& S_{0}^{4}=\left\{x_{4}, x_{5}, x_{6}, x_{7}, x_{8}, x_{9}\right\}, \\
& S_{1}^{4}=\left\{x_{1}, x_{2}, x_{3}\right\} ;
\end{aligned}
$$

45 degrees (forward)

$$
\begin{aligned}
& S_{0}^{5}=\left\{x_{1}, x_{2}, x_{3}, x_{4}, x_{5}, x_{7}\right\}, \\
& S_{1}^{5}=\left\{x_{6}, x_{8}, x_{9}\right\} ;
\end{aligned}
$$

45 degrees (reverse)

$$
\begin{aligned}
& S_{0}^{6}=\left\{x_{3}, x_{5}, x_{6}, x_{7}, x_{8}, x_{9}\right\}, \\
& S_{1}^{6}=\left\{x_{1}, x_{2}, x_{4}\right\} ;
\end{aligned}
$$

135 degrees (forward)

$$
\begin{aligned}
& S_{0}^{7}=\left\{x_{1}, x_{2}, x_{3}, x_{5}, x_{6}, x_{9}\right\}, \\
& S_{1}^{7}=\left\{x_{4}, x_{7}, x_{8}\right\} ;
\end{aligned}
$$

135 degrees (reverse)

$$
\begin{aligned}
& S_{0}^{8}=\left\{x_{1}, x_{4}, x_{5}, x_{7}, x_{8}, x_{9}\right\}, \\
& S_{1}^{8}=\left\{x_{2}, x_{3}, x_{6}\right\} .
\end{aligned}
$$

After defining the edge directions, formula (17) is applied to calculate the level of the vector of each pixel that belongs to an edge:

$$
\operatorname{Edge}(c)=\min \left(\frac{\left\|m_{0}-m_{1}\right\|}{w}, 1\right) .
$$

Edge $(c)$ is the level of possible edge direction of sets $S_{0}^{c}$ and $S_{1}^{c} ; c=1,2,3,4,5,6,7,8 . m_{0}$ and $m_{1}$ are, respectively, the mean values of the pixel vectors of sets $S_{0}^{c}$ and $S_{1}^{c}$. The threshold value $w$ is adjusted according to the level of fineness of edge assessment. The threshold value here is set in accordance with experimental results. Presently, it is set at 30. Next, we use formulas (19) and (20) to figure out the maximum edge magnitude of the pixel point and its corresponding edge direction,

$$
\begin{aligned}
E=\left[E_{x, y}\right], & x=2,3, \ldots, L-1 ; \\
y & =2,3, \ldots, N-1, \\
D=\left[D_{x, y}\right], & x=2,3, \ldots, L-1 ; \\
y & =2,3, \ldots, N-1 .
\end{aligned}
$$

$L$ and $N$, respectively, represent the length and width of the image,

$$
\begin{gathered}
E_{x, y}=\max _{c \in\{1,2,3,4,5,6,7,8\}}(\operatorname{Edge}(c)), \\
D_{x, y}=\operatorname{Arg}\left(\max _{c \in\{1,2,3,4,5,6,7,8\}}(\operatorname{Edge}(c))\right) .
\end{gathered}
$$

If Edge $(c), c=1,2,3,4,5,6,7,8$ are the same maximum value with different directions, $D_{x, y}$ would be assigned any one of the directions with maximum value. To assess with more accuracy whether each pixel point falls on an edge, the possibility that the pixel point in question and the neighboring pixel points belong to edges should be taken into account,

$$
\begin{gathered}
\text { If } D_{x, y}=1 \text { or } 2, D E_{x, y}=\frac{1}{3}\left(E_{x-1, y}+E_{x, y}+E_{x+1, y}\right), \\
\text { If } D_{x, y}=3 \text { or } 4, D E_{x, y}=\frac{1}{3}\left(E_{x, y-1}+E_{x, y}+E_{x, y+1}\right), \\
\text { If } D_{x, y}=5 \text { or } 6, \quad D E_{x, y}=\frac{1}{3}\left(E_{x-1, y-1}+E_{x, y}+E_{x+1, y+1}\right), \\
\text { If } D_{x, y}=7 \text { or } 8, D E_{x, y}=\frac{1}{3}\left(E_{x+1, y-1}+E_{x, y}+E_{x-1, y+1}\right) .
\end{gathered}
$$

After calculating all the pixels connected into edges, the edge magnitude $D E_{x, y}$ of the entire image is processed as shown in formula (22) to obtain the magnitude of nonedges and facilitate further selection of seeds and evaluation of the region growing sequence,

$$
N D E_{x, y}=1-D E_{x, y} .
$$

In the first half of Section 3.2, the ratio of pixels in the entire image that belong to edges is calculated. Then, formula (23) is applied to calculate the similarity between all the pixel points and their neighboring pixel points. Then, the maximum similarity value 1 is deducted from the result of calculation so that the larger the similarity is, the larger the value will be. $\mathbf{x}_{n}$ stands for the vector of the central point in the mask and $\mathbf{x}_{\text {mean }}$ is the mean value,

$$
S_{x, y}=1-\frac{1}{9} \sum_{n=1}^{9} \min \left(\frac{\left\|\mathbf{x}_{n}-\mathbf{x}_{\text {mean }}\right\|}{w}, 1\right) \text {, }
$$

where $\mathbf{x}_{n}, n=1,2, \ldots, 9$, and $\mathbf{x}_{\text {mean }}$ are nine pixels in the sliding window whose center is $\mathbf{x}_{x, y}$ and their mean of vectors, respectively.

The initial seed should meet the requirement that its pixel point and the neighboring pixel points are highly similar [12] and it should not be on the edge [13]. The standard for selection of the initial seed is as shown in

$$
\text { If } \min \left(N D E_{x, y}, S_{x, y}\right) \geq \mathrm{Th}, \quad \text { then } \mathbf{x}_{x, y} \text { is a seed, }
$$

where Th is a threshold.

If the similarity $S_{x, y}$ between the nonedge magnitude $N D E_{x, y}$ of a pixel point in question and the neighboring region is larger than a certain threshold value Th, it means the pixel point is a seed of interest. The setting of the threshold value Th is modified according to the level of noise in the image.

3.2. Region Growing. The similarity of the pixel vectors of all the neighboring seeded regions is calculated with formula (25) and the results are recorded in list $M$ in ascending order. 
Pixels with the highest similarity are given priority to grow to the neighboring region and the representative values $\mathbf{R}_{z}$. $\mathbf{R}_{z},=1,2, \ldots, z$ are updated. $z$ stands for the number of seeded regions and $\mathbf{R}$ is the mean value of the vectors of all the pixels in a certain seeded region,

$$
d=\min _{z=1,2, \ldots, J}\left(\frac{\left\|X_{x, y}^{M}-\mathbf{R}_{z}\right\|}{w}, 1\right) .
$$

$X_{x, y}^{M}$ represents the vector of the pixel in the $M$ list used for growing and $d$ is the smallest fuzzy similarity between pixel $X_{x, y}^{M}$ and $J$ number of neighboring seeded regions. If the pixel used for growing is near the edge of two neighboring seeded regions, classification is not as easy and this is likely to cause large changes to the representative values (mean values) of the seeded regions; after completing the calculation with formula (25), we multiply similarity $d$ with the edge magnitude $D E_{x, y}$ of the said pixel point, as shown in formula (26), before saving the results in $M$ in ascending order to allow pixels with the smallest similarity and smaller edge magnitude to have the priority to grow,

$$
d_{M}=d \cdot D E_{x, y}
$$

After the fuzzy similarity computation is completed, it is impossible to determine which of the many seeded regions should be given priority to grow. We therefore extract the smallest similarity value resulting from the calculation using formulas (25) and (26) and saved in the $M$ list as well as the regions with the largest similarity values in the $M$ list, $\mathbf{A}_{1}, \mathbf{A}_{2}, \ldots, \mathbf{A}_{m} . \mathbf{A}$ is the set of the pixel vectors of the seeded regions; $m$ refers to multispectrum space; $m=$ $\left[m_{1}, m_{2}, \ldots, m_{B}\right]^{T}$ and $B$ is the maximum number of spectrums (five spectrums in the MR images used in the experiments). Peak detection [14] is applied for further analysis, as shown in formulas (31) (34), to find out which of the regions has the largest quantity (peak value) of pixel tissues and this quantity is adopted as the new representative value of the region. The similarity between these seeded regions and the pixels used for growing is calculated anew. The steps of peak value calculation are as follows.

Step 1. According to the spectrum, the histogram $T_{B}$ indicating the number of pixels is established for each of these regions. $T_{B}$ is the histogram for the number $B$ of a certain region $\mathbf{A}_{m}$.

Step 2. The histogram $T_{B}$ established according to the spectrum of each region is smoothened, as shown in

$$
T_{B}(i)=\frac{(c(i-2)+c(i-1)+c(i)+c(i+1)+c(i+2))}{5} .
$$

$i$ stands for the pixel value of a certain region $\mathbf{A}$ in spectrum $B$ and the range of $i$ is $2 \leq i \leq L-3 . L$ is the maximum number of pixels in the whole region.
Step 3. Next, we figure out the peak value $P_{B}$ of the histogram $T_{B}$ of each spectrum in region $\mathbf{A}$, as shown in

$$
P_{B}=\left(i, T_{s}(i)\right) \mid T_{s}(i)>T_{s}(i-1), \quad T_{s}(i)>T_{s}(i+1) .
$$

The range of $i$ is $1 \leq i \leq L-2$ and $P_{B}$ is the peak value of a certain histogram $T_{B}$ of a certain region $\mathbf{A}$. Lastly, we will extract all the peak values of $\mathbf{A}_{1}, \mathbf{A}_{2}, \ldots, \mathbf{A}_{m}$ from all the spectrums and label them as $\mathbf{P k}_{1}, \mathbf{P k}_{2}, \ldots, \mathbf{P} \mathbf{k}_{m}$. $\mathbf{P k}$ is defined as the peak value of each spectrum in a certain region $\mathbf{A}$ already obtained and this peak value is regarded as the new representative value of the said seeded region. Next, formula (29) is applied to recalculate and solve the problem of being unable to assess which seeded region should grow first as a result of the calculation with formula (29) that has created the same difference between the pixel point used for growing and a number of seeded regions $\mathbf{A}_{1}, \mathbf{A}_{2}, \ldots, \mathbf{A}_{m}$. Consider

$$
\operatorname{Pd}=\min _{z=1,2, \ldots, J}\left(\left\|X_{x, y}^{M}-\mathbf{P k}_{z}\right\|\right) .
$$

$X_{x, y}^{M}$ represents the pixel vector $\mathbf{x}_{x, y}$ used for growing and $\mathrm{Pd}$ is the calculated peak value difference between pixel $X_{x, y}^{M}$ and $J$ number of neighboring seeded regions.

3.3. Region Merging. When using conventional SRG, region merging is conducted when the shortest distance (difference) between the pixel mean values of the regions is smaller than a certain threshold value. As a result, the final number of regions segmented is unknown. To prevent excessive merging of regions, we use the standard deviation target generation process $[1,15]$ to conduct region merging. The steps of the algorithm are as follows.

Step 1. Supposing the target is to merge the content of the entire image into $C$ number of tissue region types to form GM, WM, and CSF tissue regions, we first randomly create an initial target point $t_{0}$ pixel vector and use it as the center to calculate the pixel mean value $\mu_{0}$ of the neighboring $3 \times 3$ mask.

Step 2. The standard deviation SD of the entire image is calculated and the result is divided by the largest standard deviation of the entire image to conduct regularization, as shown in

$$
\mathrm{SD}=\frac{\mathrm{SD}}{\mathrm{SD}_{\max }} .
$$

Step 3. Next, it is supposed that the distribution of factual data is normal and high standard deviation should exist between the pixel information of different tissue regions (GM, WM, and CSF). formula (31) is then applied to calculate the Gaussian similarity $S_{c}$ of the entire image which is $L \times N$ in size. Consider

$$
S_{c}(x, y)=1-\operatorname{Gau}(x, y) .
$$

Gau stands for the Gaussian function Gau $=e^{-\left(I_{x, y}-v_{t}\right)^{2} / 2 \sigma^{2}} /$ $\sqrt{2 \pi \sigma^{2}}$ in which $u_{t}$ is the mask mean value of the target 


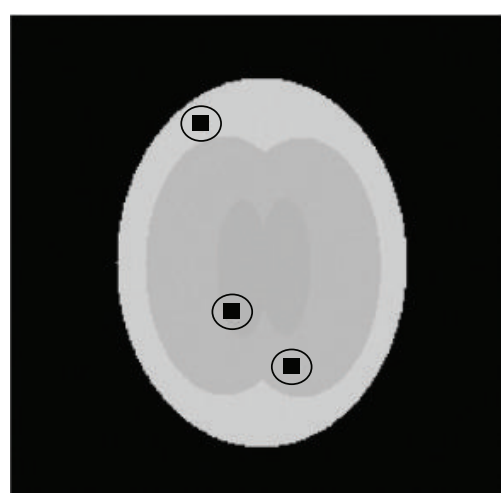

(a) $20 \mathrm{~dB}$

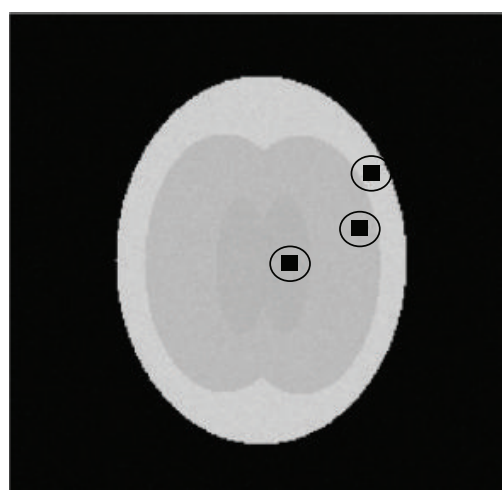

(c) $10 \mathrm{~dB}$

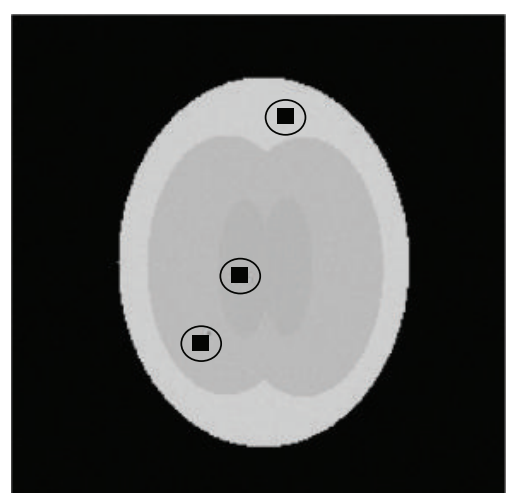

(b) $15 \mathrm{~dB}$

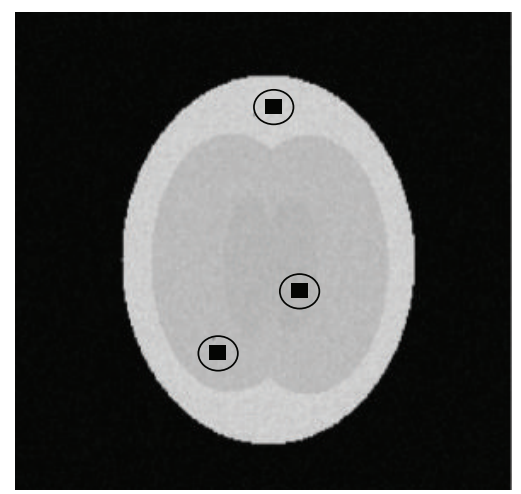

(d) $5 \mathrm{~dB}$

FIGURE 6: GM, WM, and CSF tissues selected with SDTGP from elliptical simulated brain images under different signal-to-noise ratios.

point and $\sigma$ is the standard deviation obtained earlier. When calculating the Gaussian similarity between the entire image and the target point in question, we search the entire image for the pixel point vector that has the largest difference from the target point in question and use it as the new target point $t_{c+1}$. The Gaussian similarity is calculated with formula (31) and larger Gaussian similarity is defined as smaller Gaussian similarity to facilitate the search for the next pixel with the largest difference to be the new target point. To prevent ending up with the same target point located previously when searching for the new target point, we apply formula (32) to conduct cumulative multiplication on the result of Gaussian similarity obtained from the current search throughout the entire image and that of the previous target point established and acquire $F_{c}$. The calculation is repeated until the target value of the expected amount is obtained and the target generation process algorithm is completed. Figures 6, 7, and 8 show the results of use of the target generation processing of brain MR images,

$$
F_{c}(x, y)=\frac{\prod_{k=1}^{c} S_{k}(x, y)}{\mathrm{SD}} .
$$

After completion of the SDTGP, formula (33) is applied to conduct similarity calculation on the three tissue characteristics (GM, WM, and CSF) generated with the SDTGP from all the regions in the entire image. The seeded region with the largest similarity is extracted and all the pixels therein are marked as the tissue region with the largest similarity. In the end, the final merging results are presented, respectively: GM, WM, and CSF,

$$
L R_{q}=\operatorname{Arg}\left(\min _{k \in\{1,2, \ldots, h\}}\left(\left\|R_{q}-\bar{D}^{k}\right\|\right)\right) ;
$$

$\bar{D}^{k}, k=1,2, \ldots, h$, represents the mean values of the pixel vectors of the target point (GM, WM, and CSF) tissues extracted with the SDTGP as mentioned earlier. $R_{q}, q=1$, $2, \ldots, Q$, stands for all the marked regions in the entire image. $L R_{q} \in\{1,2, \ldots, h\}$ is the number of pixel clusters in region $R_{q}$.

\section{Experiment Results}

To evaluate the quality of the classification results, we adopt a receiver operating characteristic (ROC) [11] to perform the analysis. Although the correctness and errors in the classification can be identified with the naked eye, by using ROC principles, we are able to calculate them to facilitate further examination. To perform ROC evaluation, the ground truth, also called standard classification result, of each spectrum has 


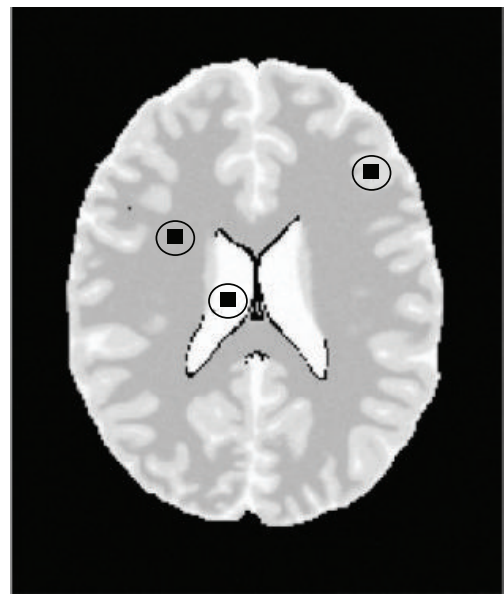

(a) $20 \mathrm{~dB}$

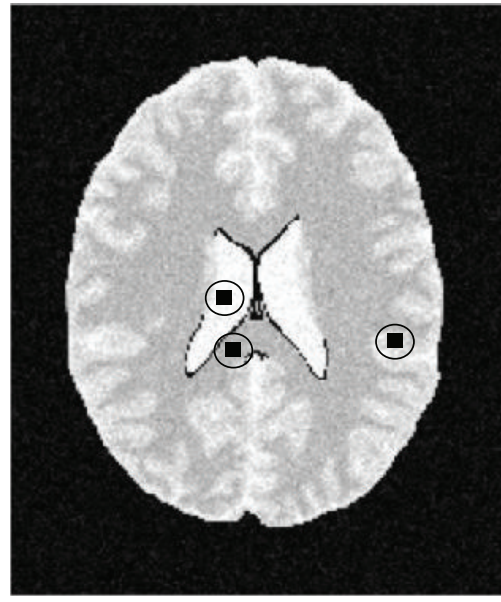

(c) $10 \mathrm{~dB}$

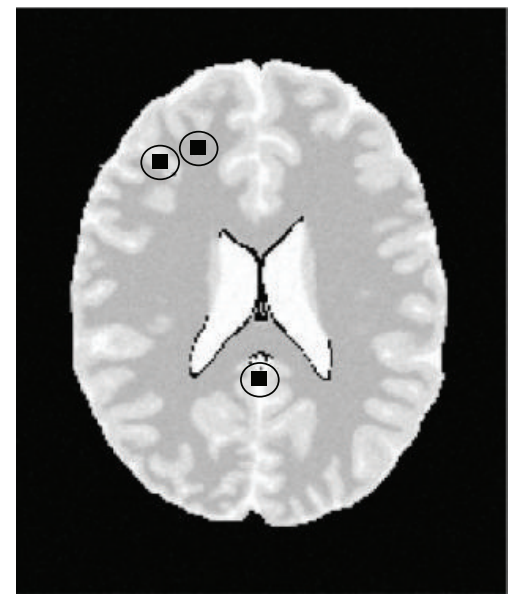

(b) $15 \mathrm{~dB}$

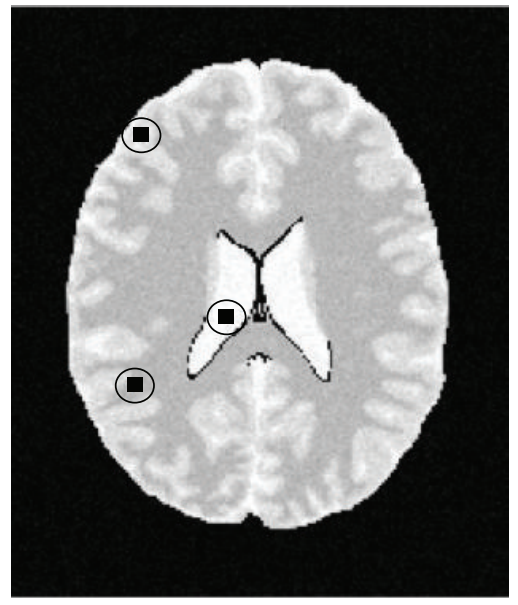

(d) $5 \mathrm{~dB}$

Figure 7: GM, WM, and CSF tissues selected with the SDTGP from BrainWeb's brain images under different signal-to-noise ratios.

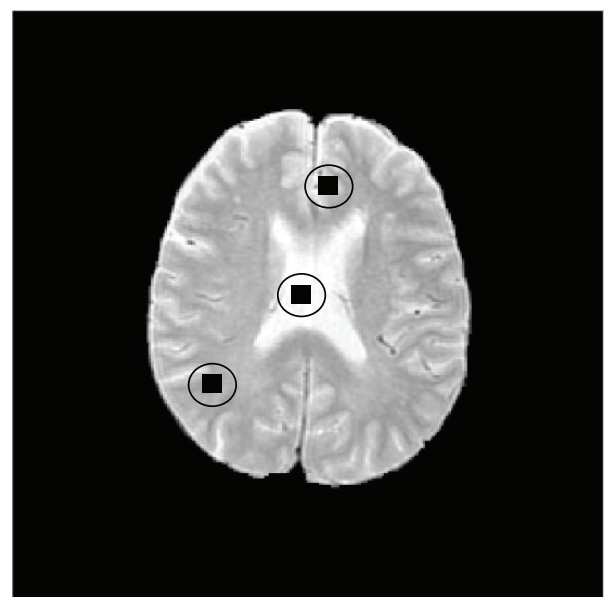

FIGURE 8: GM, WM, and CSF tissues selected with the SDTGP from real brain images under different signal-to-noise ratios. 


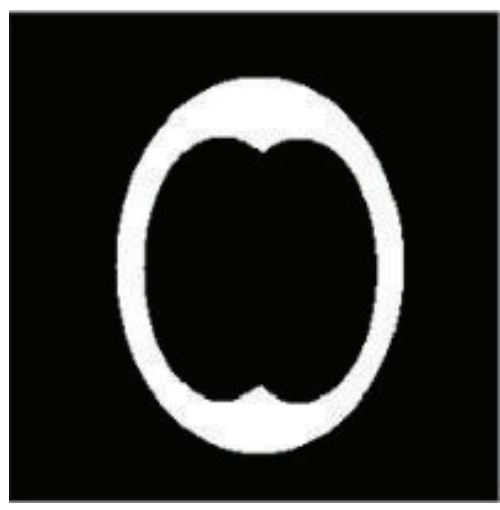

(a) GM

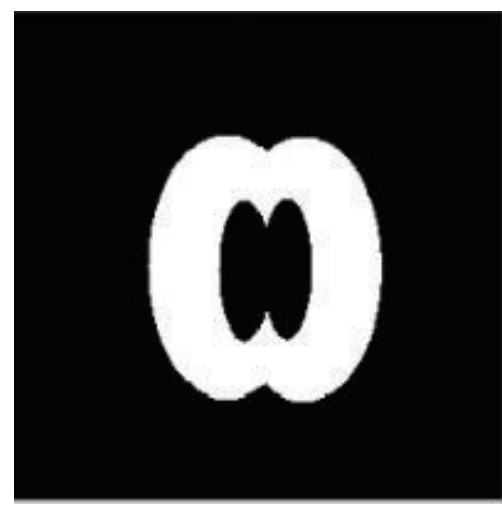

(b) WM

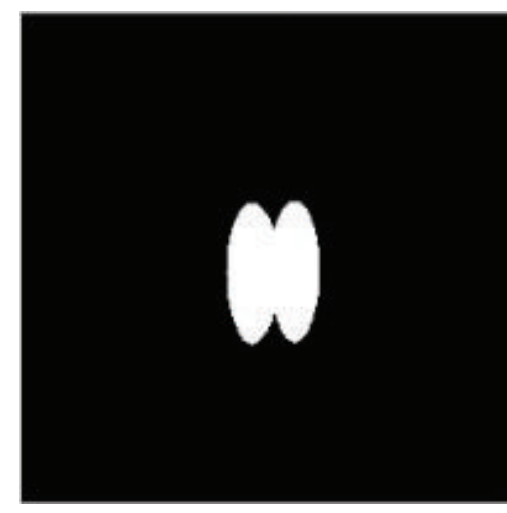

(c) CSF

FIGURE 9: FISRG classification results of elliptical simulated brain MR images (SNR $=20 \mathrm{~dB}$ ).

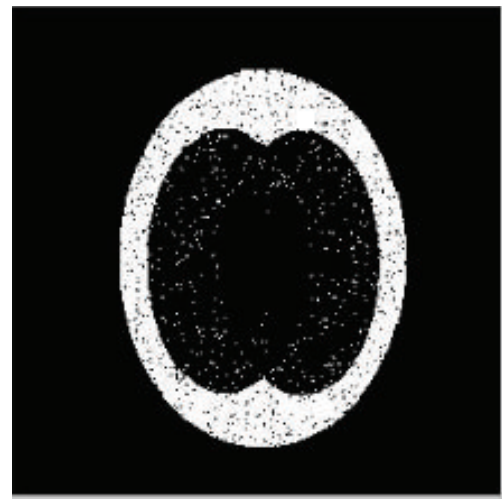

(a) GM

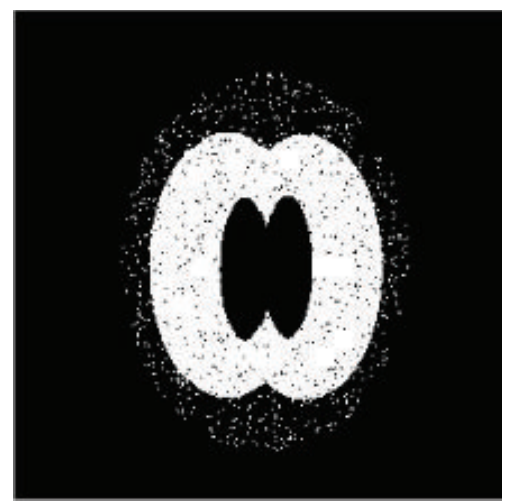

(b) WM

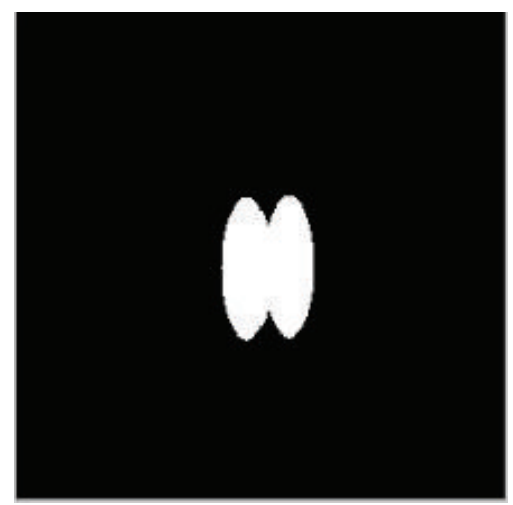

(c) $\mathrm{CSF}$

FIGURE 10: FISRG segmentation results of elliptical simulated brain MR images ( $\mathrm{SNR}=5 \mathrm{~dB}$ ).

to be matched. The ROC calculation of the detection rate $R_{D}\left(d_{i}\right)$ and false rate $R_{F}\left(d_{i}\right)$ is as follows:

$$
\begin{gathered}
R_{D}\left(d_{i}\right)=\frac{N_{D}\left(d_{i}\right)}{N\left(d_{i}\right)}, \\
R_{F}\left(d_{i}\right)=\frac{N_{F}\left(d_{i}\right)}{N-N\left(d_{i}\right)} ;
\end{gathered}
$$

$d=\left\{d_{1}, d_{2}, \ldots, d_{i}\right\}, i=1, \ldots, k$ and $k=3 . d_{i}$ is the number $i$ element in $d$. They, respectively, represent the GM, WM, and CSF. $N$ is the total number of the three types of tissues. $N\left(d_{i}\right)$ is the number of each type of tissues there should be. Each type of tissues takes up a different percentage in the image. The calculation of the total detection rate $R_{D}$ and total false rate is as follows:

$$
R_{D}=\sum_{i=1}^{k} R_{D}\left(d_{i}\right) p\left(d_{i}\right),
$$

$$
\begin{aligned}
& R_{F}=\sum_{i=1}^{k} R_{F}\left(d_{i}\right) p\left(d_{i}\right), \\
& p\left(d_{i}\right)=\frac{N\left(d_{i}\right)}{\sum_{i=1}^{k} N\left(d_{i}\right)} .
\end{aligned}
$$

$R_{D}$ is the total detection rate and $R_{F}$ is the total false rate $(k=3)$. Since the percentage of the number of each tissue type is different, each tissue type is regularized to $[0,1]$ with $p\left(d_{i}\right)$ by using a formula. The segmentation results of GM, WM, and CSF are as shown in Tables 1, 2, 3, and 4. The segmentation results are as shown in Figures 9, 10, 11, and 12 and Figure 13 is the segmentation result of the real image.

The total detection rates in Tables 5 and 6 indicate that the results of FISRG under $5 \mathrm{~dB}$ are apparently better than those of FAST and $K$-means, enough to prove that FISRG is better than the two other algorithms in noise immunity. Therefore, the classification capacity of FISRG is rather outstanding. 


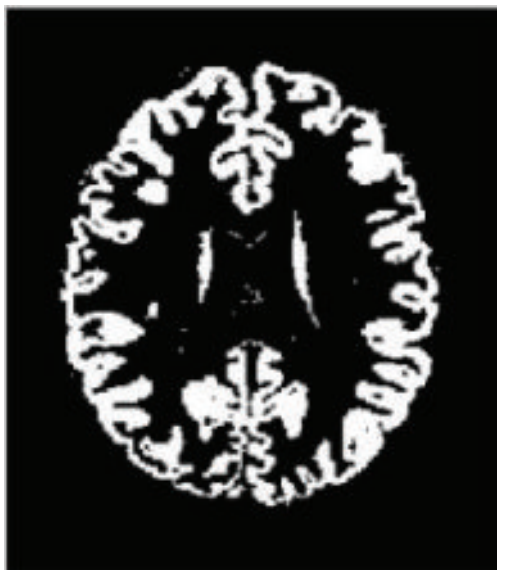

(a) GM

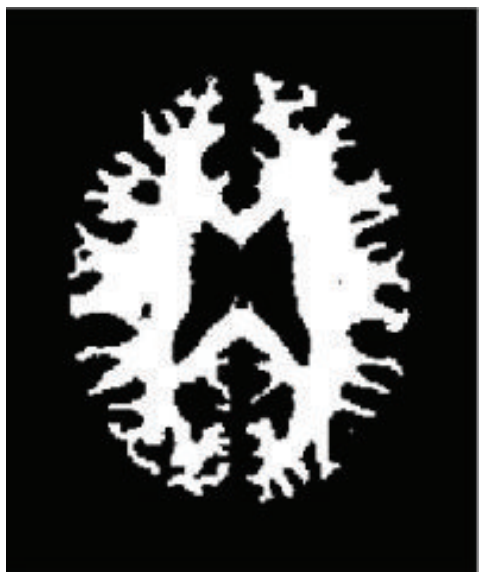

(b) WM

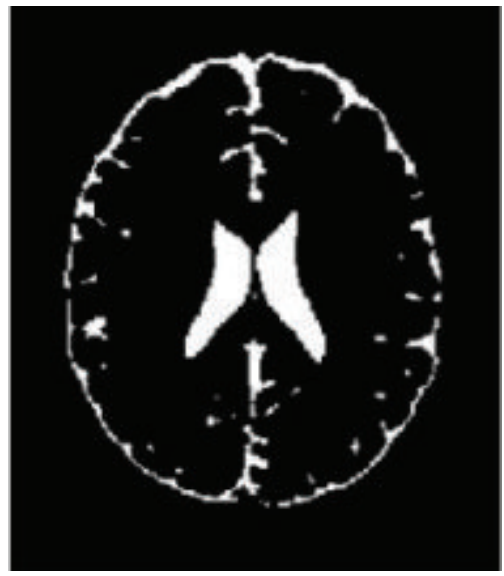

(c) $\mathrm{CSF}$

FIGURE 11: FISRG segmentation results of brain images from BrainWeb (SNR $=20 \mathrm{~dB})$.

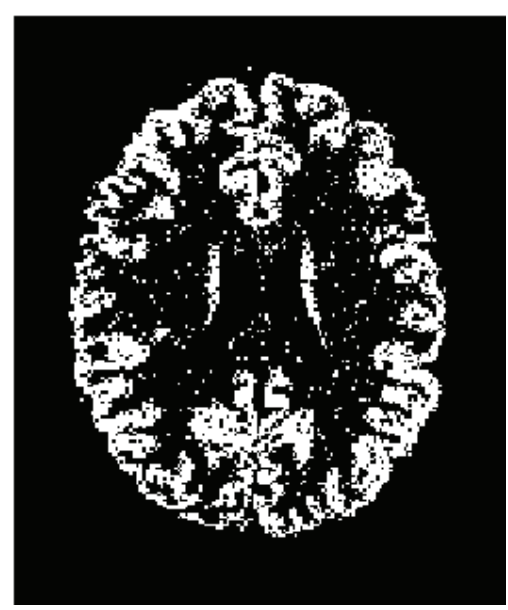

(a) GM

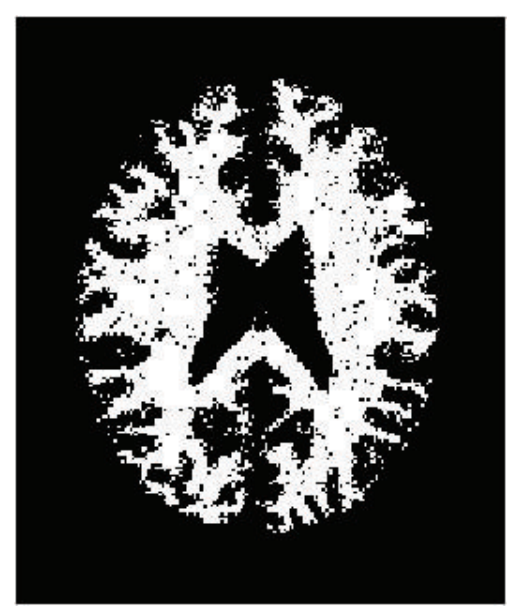

(b) WM

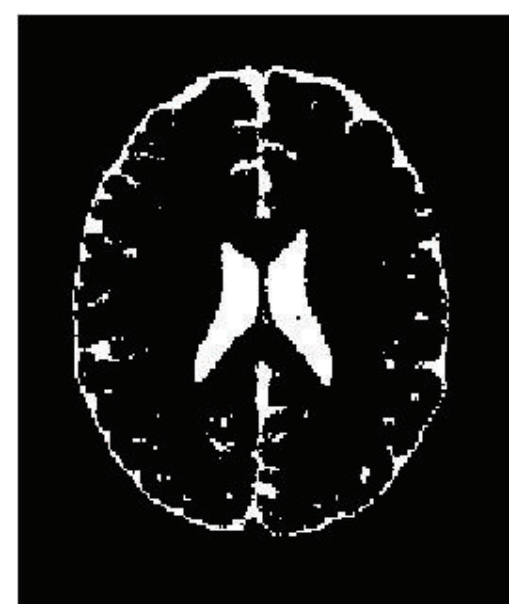

(c) CSF

FIGURE 12: FISRG segmentation results of brain images from BrainWeb (SNR $=5 \mathrm{~dB})$.

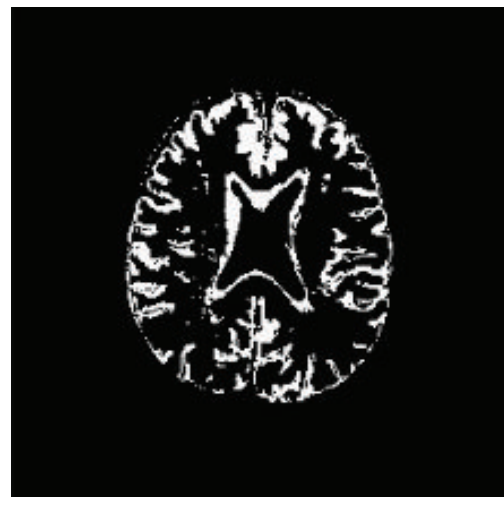

(a) GM

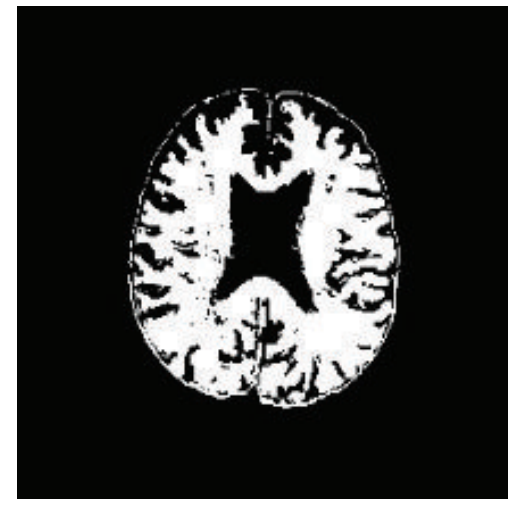

(b) WM

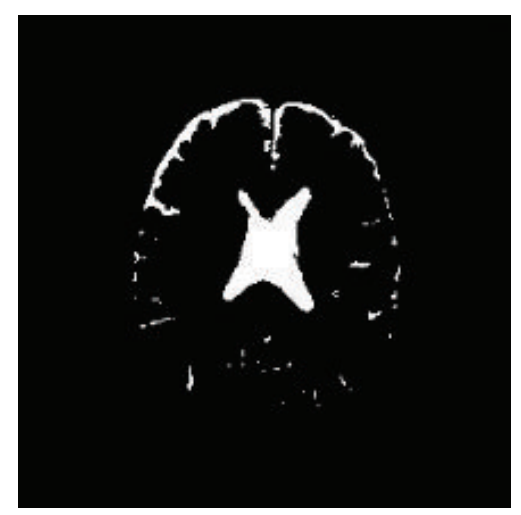

(c) $\mathrm{CSF}$

FIGURE 13: FISRG classification results of real brain MR images. 
TABLE 1: FISRG segmentation results of elliptical simulated brain MR images $(S N R=20 \mathrm{~dB})$.

\begin{tabular}{lccccccc}
\hline & $N$ & $N_{D}(d)$ & $N_{F}(d)$ & $R_{D}(d)$ & $R_{F}(d)$ & $R_{D}$ & $R_{F}$ \\
\hline GM & 9040 & 9040 & 0 & 1 & 0 & & \\
WM & 8745 & 8745 & 0 & 1 & 0 & 1 & 0 \\
CSF & 3282 & 3282 & 0 & 1 & 0 & & \\
\hline
\end{tabular}

TABLE 2: FISRG segmentation results of elliptical simulated brain MR images $(\mathrm{SNR}=5 \mathrm{~dB})$.

\begin{tabular}{lccccccc}
\hline & $N$ & $N_{D}(d)$ & $N_{F}(d)$ & $R_{D}(d)$ & $R_{F}(d)$ & $R_{D}$ & $R_{F}$ \\
\hline GM & 9111 & 8564 & 379 & 0.9400 & 0.0265 & & \\
WM & 2968 & 2968 & 0 & 1 & 0 & 0.9604 & 0.0322 \\
CSF & 11319 & 10940 & 547 & 0.9665 & 0.0453 & & \\
\hline
\end{tabular}

TABLE 3: FISRG segmentation results of brain images from BrainWeb $(20 \mathrm{~dB})$.

\begin{tabular}{lccccccc}
\hline & $N$ & $N_{D}(d)$ & $N_{F}(d)$ & $R_{D}(d)$ & $R_{F}(d)$ & $R_{D}$ & $R_{F}$ \\
\hline GM & 6552 & 6448 & 280 & 0.9841 & 0.0229 & & \\
WM & 2868 & 2708 & 14 & 0.9442 & 0.0087 & 0.9796 & 0.0129 \\
CSF & 9367 & 9247 & 90 & 0.9872 & 0.0096 & & \\
\hline
\end{tabular}

TABLE 4: FISRG segmentation results of brain images from BrainWeb (5 dB).

\begin{tabular}{lccccccc}
\hline & $N$ & $N_{D}(d)$ & $N_{F}(d)$ & $R_{D}(d)$ & $R_{F}(d)$ & $R_{D}$ & $R_{F}$ \\
\hline GM & 6552 & 5448 & 918 & 0.8315 & 0.0750 & & \\
WM & 2868 & 2671 & 225 & 0.9313 & 0.0141 & 0.8924 & 0.0722 \\
CSF & 9367 & 8646 & 879 & 0.9230 & 0.0933 & & \\
\hline
\end{tabular}

TABLE 5: Total detection rates of segmentation with FISRG, FAST, and $K$-means algorithms on elliptical simulated brain MR images.

\begin{tabular}{lcccc}
\hline & $\mathrm{SNR}=20 \mathrm{~dB}$ & $\mathrm{SNR}=15 \mathrm{~dB}$ & $\mathrm{SNR}=10 \mathrm{~dB}$ & $\mathrm{SNR}=5 \mathrm{~dB}$ \\
\hline FISRG & $100 \%$ & $100 \%$ & $99.96 \%$ & $96.04 \%$ \\
FAST & $100 \%$ & $99.99 \%$ & $99.98 \%$ & $72.48 \%$ \\
K-means & $100 \%$ & $92.90 \%$ & $92.02 \%$ & $82.86 \%$ \\
\hline
\end{tabular}

TABLE 6: Total detection rates of segmentation with FISRG, FAST, and $K$-means algorithms on MR images from BrainWeb.

\begin{tabular}{lcccc}
\hline & SNR $=20 \mathrm{~dB}$ & SNR $=15 \mathrm{~dB}$ & $\mathrm{SNR}=10 \mathrm{~dB}$ & $\mathrm{SNR}=5 \mathrm{~dB}$ \\
\hline FISRG & $97.96 \%$ & $97.25 \%$ & $95.68 \%$ & $89.24 \%$ \\
FAST & $96.24 \%$ & $95.38 \%$ & $84.85 \%$ & $74.22 \%$ \\
K-means & $94.44 \%$ & $92.17 \%$ & $88.60 \%$ & $82.47 \%$ \\
\hline
\end{tabular}

\section{Conclusion}

This paper focuses on the application of FISRG to conduct tissue segmentation research on brain MRI. Fuzzy edge detection is used to calculate the magnitude of pixel points connected into edges and apply this information to overcome problems in sequencing in region growing to make the region growing process more stable. When the same difference occurs between a pixel used for growing and a number of neighboring seeded regions during the growing and merging stages, peak detection is added to find out where in a seeded region the number of pixels is larger to establish the new similarity characteristic of the region and the pixel used for growing. During the merging stage, the standard deviation target generation process is adopted to generate the correct information about the quantity of tissues merged (GM, WM, and CSF) to overcome the inability to find out how many regions merged will lead to excessive merging or segmentation when using conventional SRG. The averages of the experimental results are extracted 100 times for analysis. FISRG proves superior to $K$-means and FAST on elliptical simulated brain images and in BrainWeb's experiments both under $20 \mathrm{~dB}$ and $5 \mathrm{~dB}$ SNR. Therefore, the classification performance, noise immunity, and stability of the FISRG algorithm prove rather outstanding.

\section{Conflict of Interests}

The authors declare that there is no conflict of interests regarding the publication of this paper.

\section{References}

[1] C. M. Wang and G. C. Lin, "Using PSO Optimize Correlation Function for MRI Classification," ILT, 2012.

[2] N. Kehtarnavaz, J. Monaco, J. Nimtschek, and A. Weeks, "Color image segmentation using multi-scale clustering," in Proceedings of the IEEE Southwest Symposium on Image Analysis and Interpretation, pp. 142-147, April 1998.

[3] T. Uchiyama and M. A. Arbib, "Color image segmentation using competitive learning," IEEE Transactions on Pattern Analysis and Machine Intelligence, vol. 16, no. 12, pp. 1197-1206, 1994.

[4] M. A. Ruzon and C. Tomasi, "Color edge detection with the compass operator," in Proceedings of the IEEE Computer Society Conference on Computer Vision and Pattern Recognition (CVPR '99), vol. 2, pp. 160-166, June 1999.

[5] P. E. Trahanias and A. N. Venetsanopoulos, "Vector order statistics operators as color edge detectors," IEEE Transactions on Systems, Man, and Cybernetics, vol. 26, no. 1, pp. 135-143, 1996.

[6] C. H. Cheng, G. C. Lin, S. W. Ju, H. C. Wang, and C. M. Wang, "Vector seeded region growing for parenchyma classification," in Proceedings of the IEEE 4th International Conference on New Trends in Information Science and Service Science (NISS '10), pp. 721-724, May 2010.

[7] Y. Deng, B. S. Manjunath, and H. Shin, "Color image segmentation," in Proceedings of the IEEE Computer Society Conference on Computer Vision and Pattern Recognition (CVPR '99), pp. 446451, June 1999.

[8] R. Adams and L. Bischof, "Seeded region growing," IEEE Transactions on Pattern Analysis and Machine Intelligence, vol. 16, no. 6, pp. 641-647, 1994.

[9] A. Mehnert and P. Jackway, "An improved seeded region growing algorithm," Pattern Recognition Letters, vol. 18, no. 10, pp. 1065-1071, 1997. 
[10] G.-C. Lin, W.-J. Wang, C.-C. Kang, and C.-M. Wang, "Multispectral MR images segmentation based on fuzzy knowledge and modified seeded region growing," Magnetic Resonance Imaging, vol. 30, no. 2, pp. 230-246, 2012.

[11] C.-C. Kang and W.-J. Wang, "Fuzzy based seeded region growing for image segmentation," in Proceedings of the Annual Meeting of the North American Fuzzy Information Processing Society (NAFIPS '09), pp. 1-5, June 2009.

[12] F. Y. Shih and S. Cheng, "Automatic seeded region growing for color image segmentation," Image and Vision Computing, vol. 23, no. 10, pp. 877-886, 2005.

[13] J. Fan, G. Zeng, M. Body, and M.-S. Hacid, "Seeded region growing: an extensive and comparative study," Pattern Recognition Letters, vol. 26, no. 8, pp. 1139-1156, 2005.

[14] K. Siang Tan and N. A. Mat Isa, "Color image segmentation using histogram thresholding-fuzzy C-means hybrid approach," Pattern Recognition, vol. 44, no. 1, pp. 1-15, 2011.

[15] G.-C. Lin, W.-J. Wang, C.-M. Wang, and S.-Y. Sun, "Automated classification of multi-spectral MR images using linear discriminant analysis," Computerized Medical Imaging and Graphics, vol. 34, no. 4, pp. 251-268, 2010. 


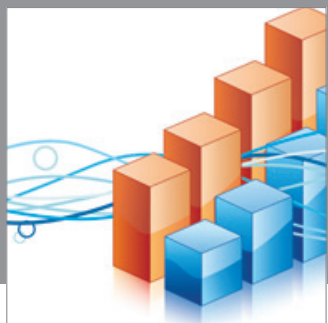

Advances in

Operations Research

mansans

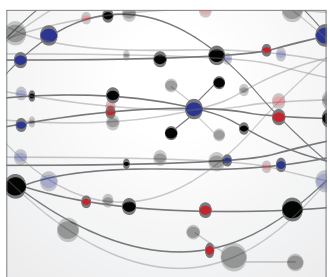

The Scientific World Journal
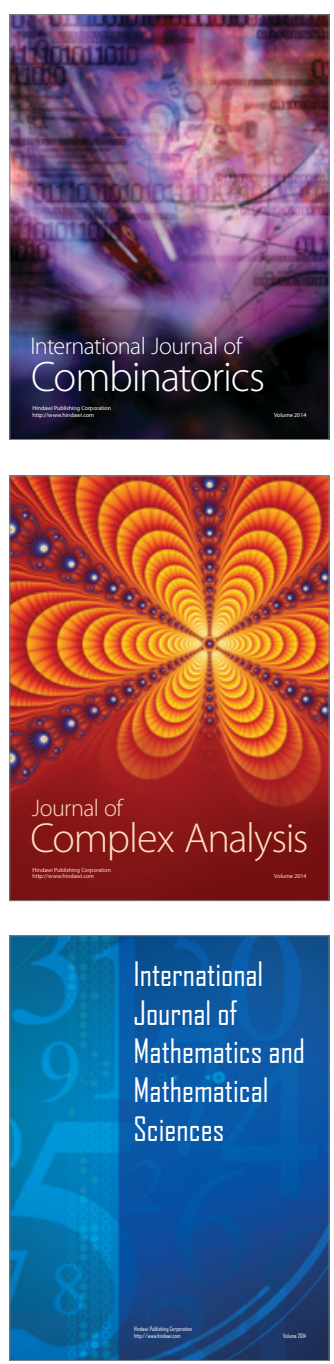
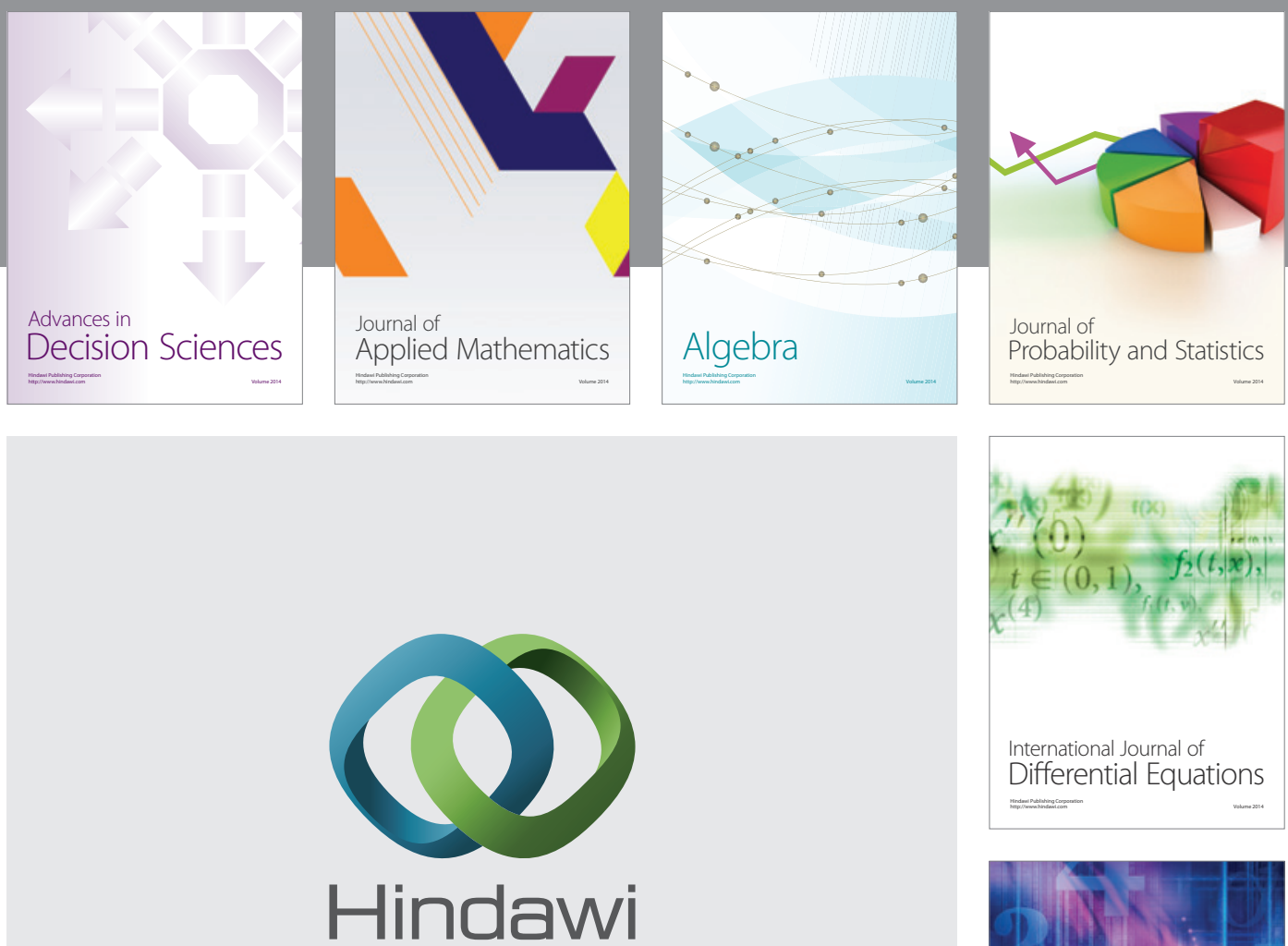

Submit your manuscripts at http://www.hindawi.com
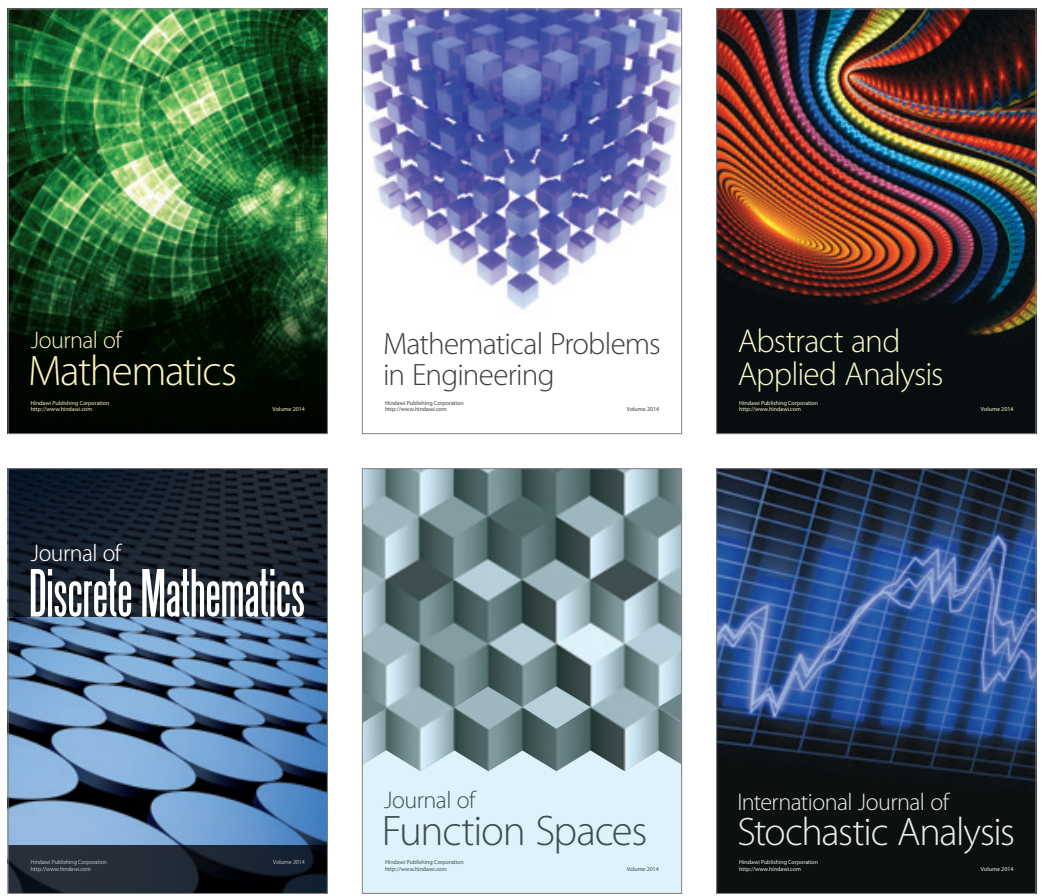

Journal of

Function Spaces

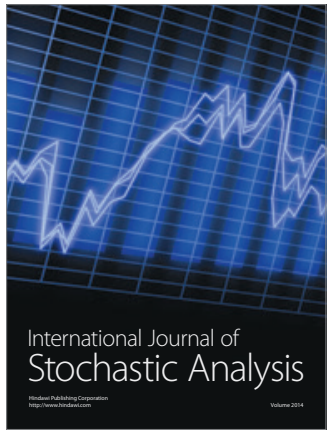

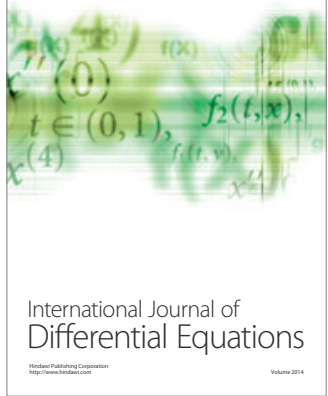
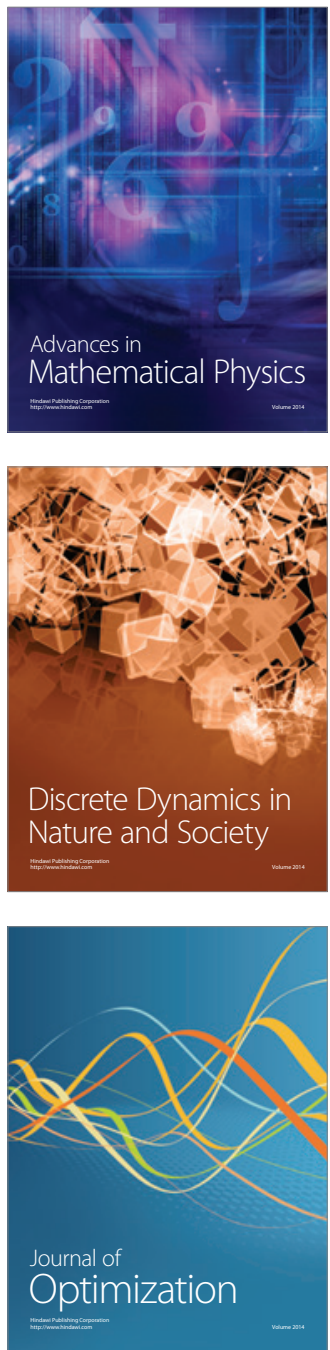